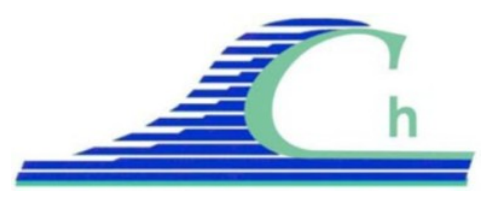

XII ${ }^{\text {èmes }}$ Journées Nationales Génie Côtier - Génie Civil

Cherbourg, 12-14 juin 2012

DOI:10.5150/jngcgc.2012.033-L_ @ Editions Paralia CFL

disponible en ligne - http://www.paralia.fr - available online

\title{
Effondrements des falaises crayeuses en Haute-Normandie
}

\author{
Pauline LETORTU ${ }^{1}$, Stéphane COSTA ${ }^{1}$, \\ Jean-Michel CADOR ${ }^{1}$, Hervé QUENOL ${ }^{2}$
}

1. Université de Caen, UFR Géographie, UMR 6554 CNRS - LETG-Caen GEOPHEN, Esplanade de la Paix, BP 5186, 14032 Caen, France.

pauline.letortu@unicaen.fr

2. Université de Rennes 2, UFR Géographie, UMR 6554 CNRS - LETG-Rennes

COSTEL, 6 avenue Gaston Berger, 35043 Rennes, France.

herve.quenol@uhb.fr

\section{Résumé :}

L'originalité de ce travail repose sur la collaboration avec l'association ESTRAN qui, depuis 2002, recense les effondrements des falaises crayeuses du linéaire côtier entre Sainte-Marguerite-sur-Mer et Bracquemont (Haute-Normandie). Cette base de données « effondrements » (331 effondrements entre 2002 et 2009) est unique en raison de la longueur de la période d'observation (8 ans) et de l'importante périodicité des levés (hebdomadaire).

La répartition spatiale et temporelle des effondrements permet de déterminer les espaces sensibles comme le secteur du Cap d'Ailly et met en évidence leur survenue toute l'année. Cependant, l'hiver est la saison la plus propice à l'érosion (aussi bien en volume effondré qu'en nombre d'effondrements), suivi de l'automne et du printemps. L'été est la période la moins propice sans être exempte d'effondrements massifs.

L'analyse statistique utilisée (A.C.P.) souligne que ces mouvements sont le produit d'une combinaison de facteurs. Les facteurs marins semblent prépondérants en hiver puis à l'automne tandis qu'en été puis au printemps, les facteurs continentaux prédominent, générant des effondrements souvent moins nombreux et moins volumineux. L'analyse statistique nous permet également de proposer des éléments de réponses quant aux périodes de retour de ces mouvements selon leurs volumes et leurs répartitions spatiales (ajustement à la loi de puissance).

Cette approche quantitative utilisée n'est pertinente que si elle est couplée à une approche géomorphologique afin de distinguer les facteurs responsables du déclenchement des effondrements.

\section{Mots-clés :}

Erosion côtière - Falaises crayeuses - Haute-Normandie - Effondrements - Processus Géomorphologie 


\section{Coastal chalk cliff falls in Upper Normandy}

\section{Abstract:}

The study is carried out in collaboration with the non-profit corporation, ESTRAN. Thanks to this original partnership, we have a remarkable and unique database describing 331 coastal chalk cliff falls from 2002 to 2009 along the chalk cliff line from Sainte-Marguerite-sur-Mer to Bracquemont (Upper Normandy, France). This database has numerous advantages: a long period of observation (8 years) and a high-periodicity of survey (every week).

Spatial and temporal distributions of cliff falls highlight sensitive areas as Ailly cape and that cliff falls occur all year long. However, winter is the main season of cliff falls occurrence (in fallen volume and number of falls), then autumn and spring. Summer is not a season prone to cliff falls but some massive falls might occur.

The statistical approach used in this study (P.C.A.) emphasizes that cliff fall is the result of a combination of processes. Marine processes seem mainly responsible for falls in winter, then in autumn and sub-aerial weathering processes seem predominant in summer, then in spring, but falls are less numerous and less massive. Statistical approach (fitting to a power law) provides answers about return periods of falls according to fallen volumes and spatial distributions.

This statistical approach used in this study is only relevant if it is linked with geomorphologic approach in order to distinguish factors that trigger the fall.

Keywords: Coastal erosion - Coastal chalk cliffs - Upper Normandy - Falls Processes - Geomorphology

\section{Introduction}

Les falaises de Haute-Normandie ont principalement été étudiées à partir d'un suivi du haut de l'abrupt, dont le recul moyen a été estimé à $20 \mathrm{~cm} / \mathrm{an}$ (COSTA, 2000 ; COSTA et al., 2004). Notre travail propose un inventaire des effondrements affectant l'ensemble de l'escarpement (du pied au sommet), ce qui apporte plus d'informations sur les modalités d'évolution des abrupts et les processus potentiellement responsables des effondrements (YOUNG et al., 2009). Ce travail repose sur l'analyse des facteurs externes responsables du déclenchement des effondrements (facteurs marins et continentaux), seuls éléments quantifiables de façon homogène à l'échelle du travail envisagé. Cependant, les facteurs internes sont également importants mais les données (fracturation, karstification, lithostratigraphie) ne sont pas toujours disponibles (ou hétérogènes) sur un tel linéaire (LAIGNEL, 2003 ; DUPERRET et al., 2004, HOYEZ, 2008). Ce travail repose sur un inventaire hebdomadaire des effondrements sur une partie du littoral haut-normand, initié dès 2002.

Cette base de données considérable et unique nous permet ainsi de répondre à deux questions de recherche que sont la répartition spatiale et temporelle des effondrements, ainsi que les processus responsables du déclenchement des effondrements. 


\section{XII ${ }^{\text {èmes }}$ Journées Nationales Génie Côtier - Génie Civil \\ Cherbourg, 12-14 juin 2012}

\section{Matériel et méthodes}

La difficulté dans la recherche des facteurs responsables du déclenchement des effondrements est liée à deux contraintes majeures que sont le manque de datation précise des événements de recul sur de longs pas de temps, et le manque de données précises à haute fréquence des processus jugés prépondérants dans le déclenchement des effondrements (GENTER et al., 2004).

Le recensement, initié en 2002, effectué chaque semaine par le Service Littoral de l'association ESTRAN de Dieppe, constitue donc une base de données unique.

De 2002 à 2003, le suivi a d'abord été mis en place entre Veules-les-Roses et Le Tréport $(37,5 \mathrm{~km})$. A partir de 2005, pour des raisons d'efficacité, la section côtière a été réduite à $12 \mathrm{~km}$ entre Sainte-Marguerite-sur-Mer et Bracquemont (figure 1). Cette réduction permet de suivre la moitié de la section côtière à pied (Sainte-Marguerite-sur-Mer et Varengeville-sur-Mer) en complément de points fixes d'observation définis sur l'ensemble du linéaire.

De 2002 à 2009, le recensement compte 331 effondrements datés. C'est sur cette base qu'une approche statistique (A.C.P., ajustement à la loi de puissance) et géomorphologique (zones de départ des effondrements) ont été effectuées.

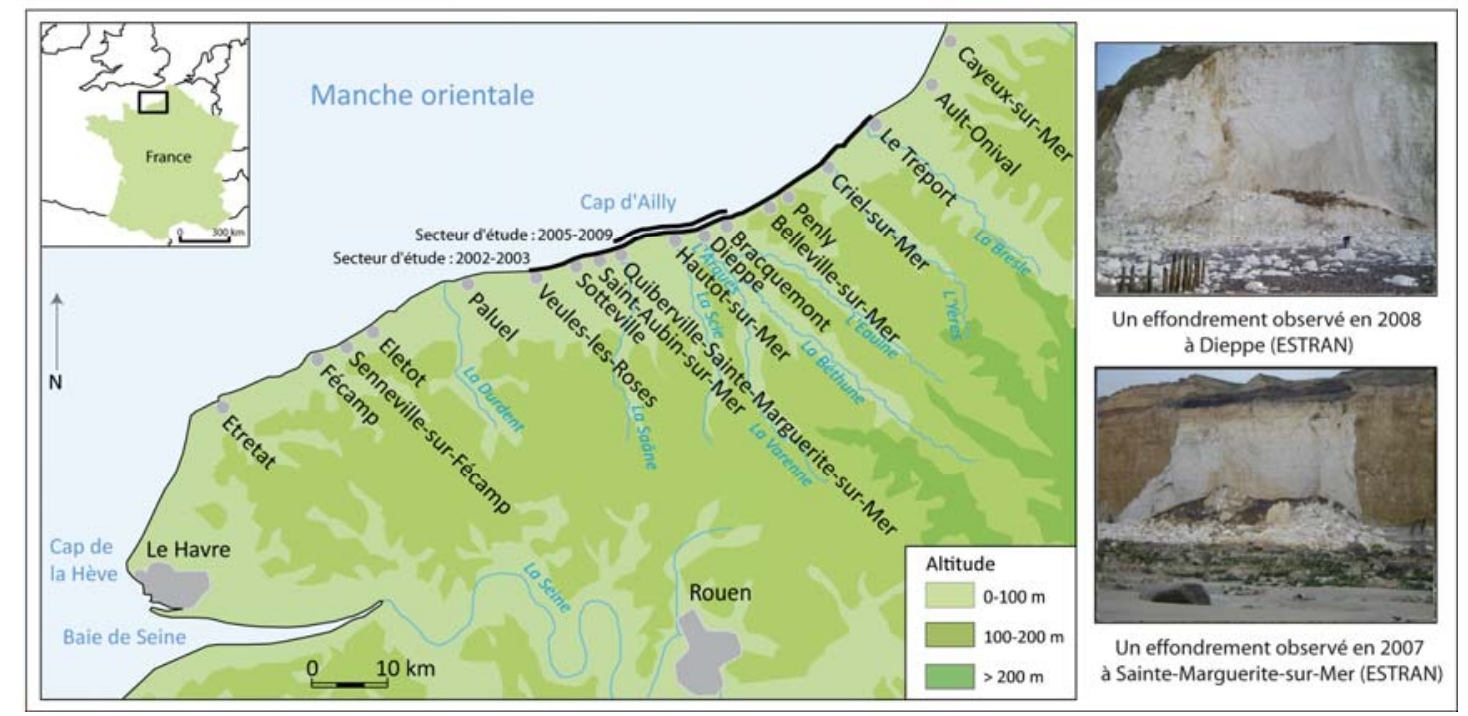

Figure 1. Présentation du secteur d'étude.

\section{Résultats}

\subsection{La répartition spatiale et temporelle des effondrements}

L'approche spatiale, au travers de la cartographie des 331 effondrements correspondant à un volume total effondré d'environ $865000 \mathrm{~m}^{3}$, permet de déterminer les espaces sensibles aux effondrements. 


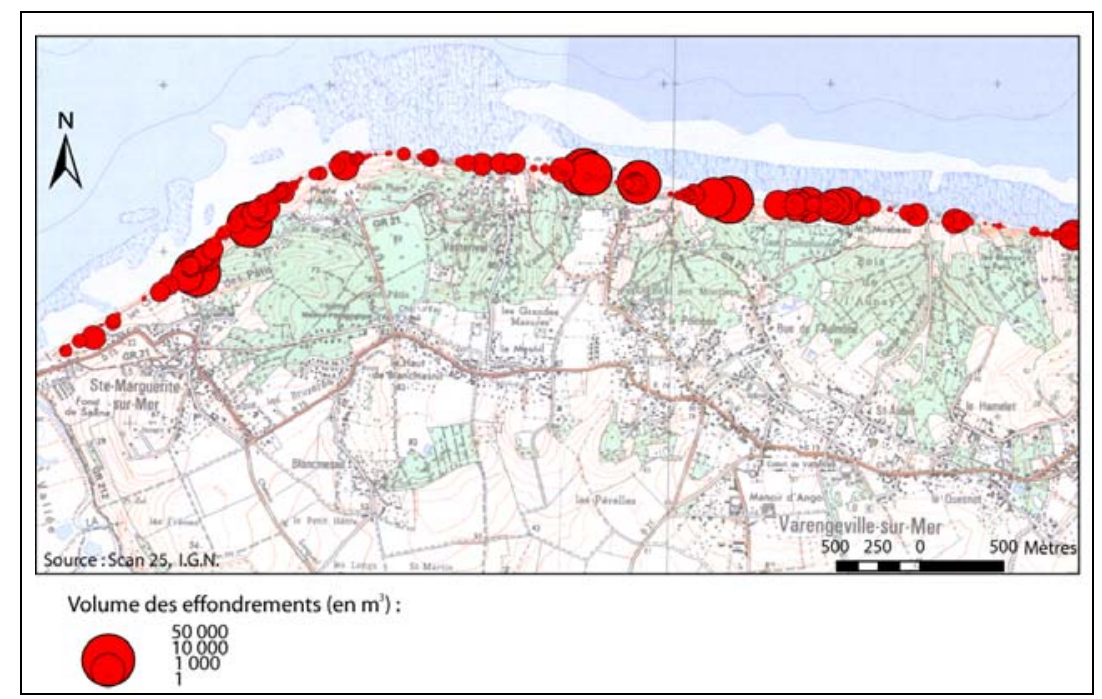

Figure 2. Effondrements recensés de 2002 à 2009 par ESTRAN sur le secteur du Cap d'Ailly (Sainte-Marguerite-sur-Mer - Varengeville-sur-Mer).

Le secteur du Cap d'Ailly est le plus sensible à la dynamique érosive (figure 2) en raison de ses caractéristiques géologiques : ces falaises sont constituées, à leur base, de craies d'âge Santonien particulièrement favorables à la météorisation (LAIGNEL, 2003), surmontées par des terrains tertiaires sableux et argileux, eux-mêmes affectés par l'existence de deux nappes aquifères perchées. Ces terrains et l'omniprésence d'eau jouent un rôle important dans le recul des falaises. La datation des effondrements permet de connaître leur répartition saisonnière. Ces derniers ont lieu toute l'année. Sans surprise, l'hiver est la saison la plus propice (aussi bien en volume effondré qu'en nombre d'effondrements), suivi de l'automne, puis du printemps. L'été est la période la moins propice mais n'est pas exempte d'effondrements massifs (figure 3).

\subsection{L'approche statistique}

Après avoir intégré à la base de données les informations relatives aux processus communément jugés responsables du déclenchement des effondrements (précipitations, gel/dégel, actions marines...), l'approche statistique adoptée s'appuie sur une Analyse en Composante Principale (A.C.P.) qui vise à étudier la structuration entre les processus et les effondrements (par décade). Cette dernière met en évidence, d'une part, que l'effondrement est le produit d'une combinaison de facteurs (absence d'un facteur unique), et d'autre part, l'opposition majeure entre les facteurs marins et continentaux, eux-mêmes affectés par une répartition saisonnière marquée. 


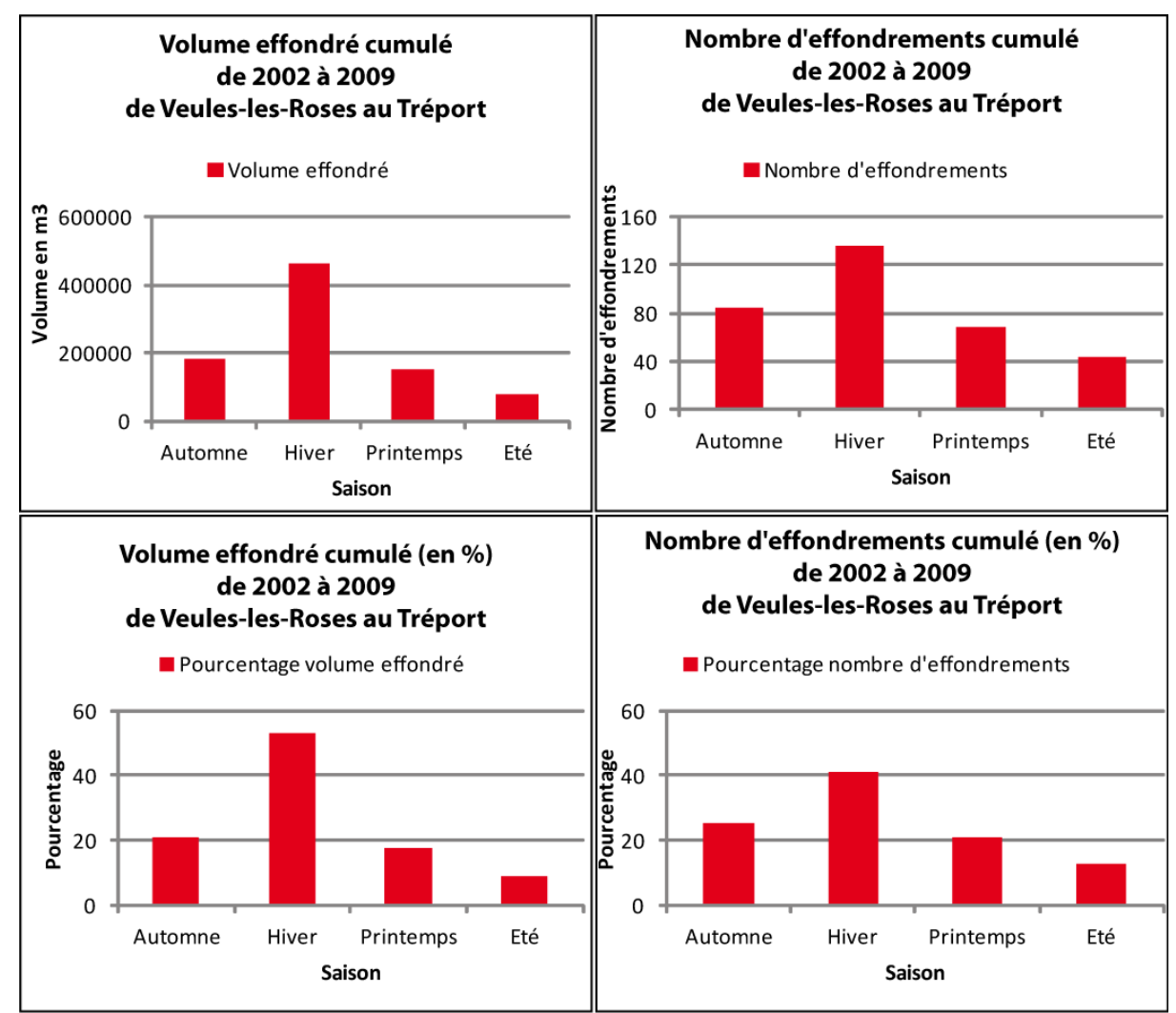

Figure 3. Répartition saisonnière des effondrements (nombre et volume cumulés).

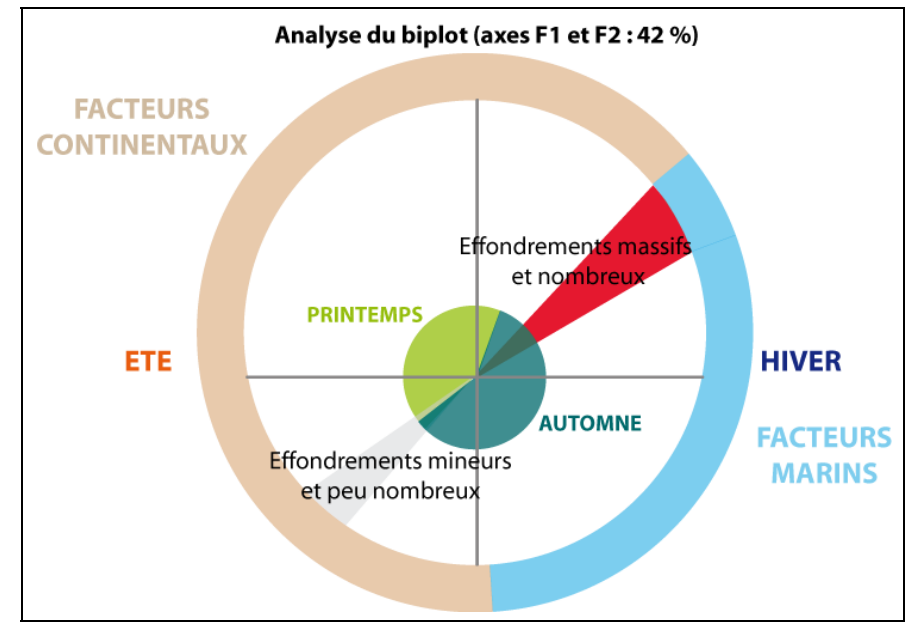

Figure 4. Interprétation de la structuration des variables de l'A.C.P. des facteurs F1-F2 et F1-F3.

Ainsi, les facteurs marins (vagues, coefficient de marée...) semblent prédominants en hiver puis à l'automne (les actions continentales demeurent, mais en plus faible proportion) tandis qu'en été, puis au printemps les facteurs continentaux (précipitations, amplitudes thermiques...) prédominent, générant des effondrements souvent moins nombreux et moins volumineux (figure 4). 
L'approche statistique utilisée (ajustement à la loi de puissance) nous permet également de proposer des éléments de réponses quant aux périodes de retour des effondrements selon leurs répartitions spatiales et leurs volumes (figure 5).

\begin{tabular}{|c|c|c|c|c|c|}
\hline Répartition spatiale & $\begin{array}{c}\text { Nombre } \\
\text { d'effondrements } \\
/ \\
\text { échantillon }\end{array}$ & $\begin{array}{c}\text { Longueur } \\
\text { de la } \\
\text { chronique } \\
\text { (années) }\end{array}$ & $\begin{array}{c}\text { Fonction } \\
\text { d'ajustement des } \\
\text { volumes effondrés } \\
\text { cumulés à la loi de } \\
\text { puissance }\end{array}$ & $\begin{array}{c}\text { Linéaire } \\
\text { de falaise } \\
\text { (km) }\end{array}$ & $\mathbf{n}_{\mathbf{1 0 0}}$ /km \\
\hline $\begin{array}{c}\text { Veules-les-Roses - } \\
\text { Le Tréport }\end{array}$ & 65 & 2 & $\mathrm{f}=407,93 \mathrm{~V}^{-0,535}$ & 37,5 & 0,9 \\
\hline $\begin{array}{c}\text { Sainte-Marguerite-sur-Mer } \\
\text { Varengeville-sur-Mer }\end{array}$ & 203 & 5 & $\mathrm{f}=379,86 \mathrm{~V}^{-0,527}$ & 6 & 5,5 \\
\hline $\begin{array}{c}\text { Hautot-sur-Mer - } \\
\text { Bracquemont }\end{array}$ & 51 & 6 & $\mathrm{f}=51,404 \mathrm{~V}^{-0,447}$ & 6 & 1,1 \\
\hline
\end{tabular}

Figure 5. Fréquence et période de retour des effondrements.

Un minimum de 5 effondrements supérieurs à $100 \mathrm{~m}^{3}$ est attendu par an $\left(\mathrm{n}_{100}\right)$ et par $\mathrm{km}$ linéaire, entre Sainte-Marguerite et Varengeville-sur-Mer (secteur du Cap d'Ailly), contre 1 effondrement de plus de $100 \mathrm{~m}^{3}$ sur les autres secteurs. Cette variabilité spatiale du nombre d'effondrements est très probablement liée aux caractéristiques morpho-structurales. Ces résultats corroborent les travaux de S. COSTA (2000, et COSTA et al., 2004) qui indiquent que, pour des raisons lithostratigraphiques, les effondrements au Cap d'Ailly sont nombreux mais peu volumineux, tandis qu'ailleurs, les effondrements sont plus massifs mais moins fréquents.

\subsection{Approche géomorphologique}

Cette approche statistique n'est pertinente que si elle est couplée à une approche géomorphologique. La zone de départ de l'effondrement est une clé de lecture évidente qui peut permettre de déterminer les possibles facteurs de déclenchement. A titre d'exemple, les effondrements en pied de falaise (même s'ils peuvent déstabiliser ensuite l'ensemble de la paroi) semblent logiquement liés aux actions marines (déferlement des vagues), alors que les mouvements du milieu et du haut de falaise seraient à mettre en relation avec les processus subaériens.

A partir de l'A.C.P., il nous a été possible de déterminer des groupes d'effondrements (par décade) induits par des actions marines prépondérantes à ce moment. La deuxième étape consiste à déterminer la répartition spatiale de ces effondrements sur l'escarpement (pied, milieu et haut de falaise) (figure 6). 


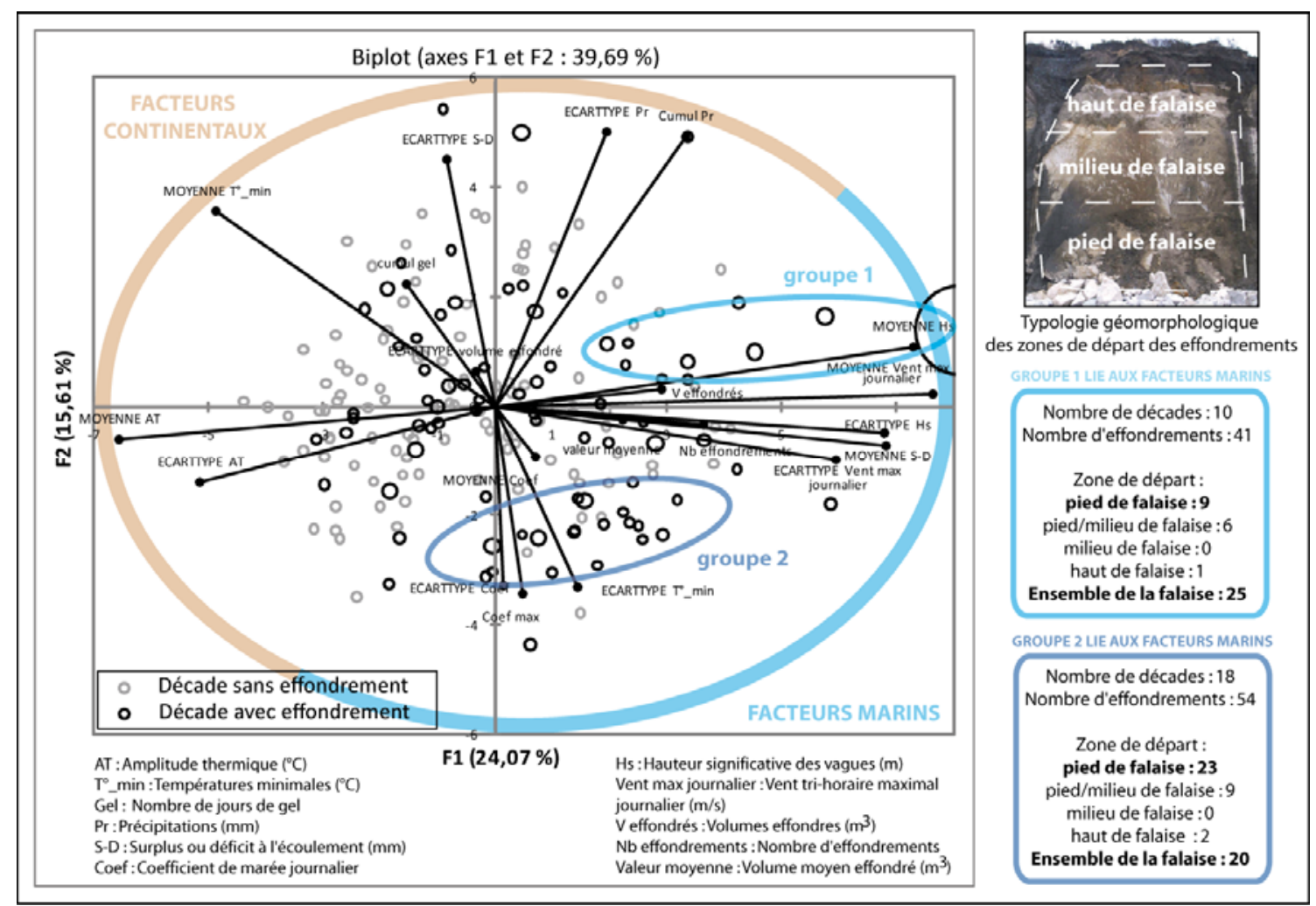

Figure 6. A.C.P. et typologie géomorphologique des zones de départ des effondrements.

Sur les deux échantillons choisis (groupes 1 et 2), soit 95 effondrements, 32 ont affectés le pied de la falaise, 15 le pied et le milieu de la falaise, et 45 l'ensemble de la falaise (figure 6). Seuls trois effondrements ont lieu en haut de l'abrupt, ce qui ne peut, a priori, être induit par les actions marines.

Cependant, là encore, en dépit de cette corrélation statistique forte, rien ne nous assure que les effondrements de pied et de pan entier de falaise soient dus exclusivement aux seules actions marines, a priori prépondérantes. En effet, les fortes agitations marines sont induites par des situations atmosphériques dépressionnaires également caractérisées par des précipitations instantanées et/ou cumulées importantes, elles aussi favorables au déclenchement des effondrements.

Pour parfaire ces premiers résultats, il convient désormais d'obtenir des informations à plus haute fréquence et spatialement encore plus précises sur les effondrements.

\section{Conclusions}

Les diverses approches (spatiale, temporelle, statistique et géomorphologique) utilisées pour l'exploitation de la base de données nous ont permis d'identifier :

- les espaces sensibles aux effondrements comme le secteur du Cap d'Ailly, mais aussi la permanence des effondrements au cours de l'année. Cependant, l’hiver est la saison 
la plus propice au recul (en volume effondré et en nombre d'effondrements), suivi de l'automne, du printemps et de l'été.

- que l'effondrement est le produit d'une combinaison de facteurs. Les facteurs marins semblent prédominants en hiver et à l'automne tandis qu'en été et au printemps, les facteurs continentaux l'emportent, générant des effondrements souvent moins nombreux et moins volumineux. L'approche statistique nous fournit aussi des périodes de retour des effondrements selon leurs répartitions spatiales et leurs volumes.

- l'importance de l'approche géomorphologique dans la définition des facteurs déclenchants. Cependant, l'identification du processus déclenchant reste complexe en raison du manque de précision temporelle de la datation et de la complexité des combinaisons de facteurs affectant le massif crayeux.

\section{Références bibliographiques}

COSTA S. (2000). Réactualisation des connaissances et mise en place d'une méthode de suivi de la dynamique du littoral haut-normand et picard. Rapport final, Préfecture de Picardie, Contrat de Plan Interrégional du Bassin de Paris (CPIBP), 103 p.

COSTA S., DELAHAYE D., FREIRE-DIAZ S., DI-NOCERA L., DAVIDSON R., PLESSIS E. (2004). Quantification of the Normandy and Picardy chalk cliff retreat by photogrammetric analysis. In: MORTIMORE R.N., DUPERRET A. (eds), Coastal chalk cliff instability, Geological Society, London, Eng. Geol. Sp., 20, pp 139-148.

DUPERRET A., GENTER A., MARTINEZ A., MORTIMORE R.N. (2004). Coastal chalk cliff instability in NW France: role of lithology, fracture pattern and rainfall. In: MORTIMORE R.N., DUPERRET A. (eds), Coastal chalk cliff instability, Geological Society, London, Eng. Geol. Sp., 20, pp 33-55.

GENTER A., DUPERRET A., MARTINEZ A., MORTIMORE R.N., VILA J.L. (2004). Multiscale fracture analysis along the French chalk coastline for investigating erosion by cliff collapse. In: MORTIMORE R.N., DUPERRET A. (eds), Coastal chalk cliff instability, Geological Society, London, Eng. Geol. Sp., 20, pp 57-74.

HOYEZ B. (2008). Falaises du Pays de Caux. Lithostratigraphie des craies turonocampaniennes. Publications des Universités de Rouen et du Havre, Mont-Saint-Aignan, $348 \mathrm{p}$.

LAIGNEL B. (2003). Caractérisation et dynamique érosive de systèmes géomorphologiques continentaux sur substrat crayeux. Exemple de l'Ouest du Bassin de Paris dans le contexte nord-ouest européen. Mémoire d'Habilitation à Diriger des Recherches, Université de Rouen, 138 p.

YOUNG A.P., FLICK R.E., GUTIERREZ R., GUZA R.T. (2009). Comparison of short-term seacliff retreat measurement methods in Del Mar, California. Geomorphology, n²112, pp 318-323. doi:10.1016/j.geomorph.2009.06.018 